\title{
LIMITE ERGODIQUE DE PROCESSUS DE DIFFUSION INFINI-DIMENSIONNELS
}

\author{
S. Relly et D. Seu
}

\begin{abstract}
We give a temporal ergodicity criterium for the solution of a class of infinite dimensional stochastic differential equations of gradient type, where the interaction has infinite range. We illustrate our theoretical result by typical examples.
\end{abstract}

\section{Introduction}

$\mathrm{Au}$ cours de ces dernières décennies, des progrès majeurs ont été faits sur l'étude du comportement asymptotique en temps de processus de diffusion sur un réseau, qui sont solutions d'équations différentielles stochastiques à valeurs dans $M^{S}$ où $M$ est un espace métrique complet et $S$ un ensemble dénombrable (infini).

Parmi les résultats les plus récents, on peut citer quand $M=\mathbb{R}$ et $S=\mathbb{Z}^{d}$, Zegarlinski (1996) qui a montré la convergence à vitesse exponentielle de la loi au temps $t$ de certaines diffusions gradients vers une mesure de gibbs, par des méthodes utilisant les semi-groupes hypercontractants et les inégalités de Log-Sobolev. Da Prato \& Zabczyk (1995) utilisent dans le cas où $M=\mathbb{R}$, ou bien $M$ espace de Hilbert, des propriétés de dissipativité d'opérateurs sur des espaces de Hilbert pour obtenir l'ergodicité du processus. Ou encore Albeverio \& al. (1997) montrent la convergence ergodique (dans $L^{2}$ ) du processus grâce aux techniques de convergence des formes de Dirichlet associées.

Keywords. Infinite dimensional diffusion, ergodicity. 
Dans tous ces travaux les interactions intervenant dans la dynamique sont à portée finie, i.e. l'équation différentielle stochastique satisfaite par chaque coordonnée de la diffusion (à valeurs dans $M$ ) ne fait intervenir qu'un nombre fini d'autres coordonnées.

Nous présentons ici, un premier exemple d'ergodicité pour des diffusions à valeurs dans $\mathbb{R}^{\mathbb{Z}^{d}}$ avec interaction à portée infinie et la technique que nous utilisons (très simple par rapport aux autres techniques précédemment citées) fait appel à une idée de Sunyach, développée élégamment par Royer (1979): Sunyach (1975) exhibe un critère d'ergodicité dans un cadre général en considérant la classe de processus dont le semi-groupe de transition a une action contractante sur l'espace des fonctions lipschitziennes.

Dans ce travail, nous considérons le système gradient stochastique suivant dans $\mathbb{R}^{\mathbb{Z}^{d}}(d \geq 1): \forall i \in \mathbb{Z}^{d}$,

$$
\begin{aligned}
d X_{i}(t) & =d B_{i}(t)-\frac{1}{2}\left(\phi_{i}^{\prime}\left(X_{i}(t)\right)+\sum_{j \neq i} \phi_{i, j}^{\prime}\left(X_{i}(t), X_{j}(t)\right)\right) d t, \quad t>0 \\
X(0) & =x \in \mathbb{R}^{\mathbb{Z}^{d}}
\end{aligned}
$$

où les $\phi_{i}, i \in \mathbb{Z}^{d}$, sont des fonctions sur $\mathbb{R}$ appelées potentiels d'interaction propres et pour $i \neq j$, les $\phi_{i, j}$ sont des fonctions sur $\mathbb{R}^{2}$ satisfaisant $\phi_{i, j}=\phi_{j, i}$, appelées potentiels d'interaction par paires; on pose, pour $x, y \in \mathbb{R}$,

$$
\phi_{i, j}^{\prime}(x, y)=\frac{\partial}{\partial x} \phi_{i, j}(x, y)
$$

$\left(B_{i}\right)_{i \in \mathbb{Z}^{d}}$ est une famille de mouvements browniens réels indépendants.

L'existence et l'unicité de la solution du système (1) dans $\mathcal{S}^{\prime}$ ont été montrées sous des hypothèses que nous adopterons (cf Doss \& Royer (1978), Shiga \& Shimizu (1980)).

Dans le paragraphe 2 , le Théorème 1 , qui est notre résultat principal, montre que sous certaines hypothèses sur $\phi_{i}$ et $\phi_{i, j}$, le processus $(X(t))_{t \geq 0}$ solution de (1) est ergodique dans un certain espace de Hilbert $E_{-r}$, que l'interaction soit à portée finie ou infinie.

Dans le paragraphe 3, on donnera deux exemples de potentiels d'interaction satisfaisant les hypothèses du Théorème 1: pour le premier, l'interaction est à portée finie et pour l'autre la portée est infinie.

Nous allons tout d'abord préciser certaines notations. 


\section{Notations.}

- $\mathbb{R}^{\mathbb{Z}^{d}}(d \geq 1)$ est l'espace d'états muni de sa filtration canonique $\left(\mathcal{F}_{\Lambda}\right)_{\Lambda}$, où $\Lambda$ décrit l'ensemble des parties finies de $\mathbb{Z}^{d} \cdot \mathcal{F}_{\Lambda}$ est donc engendrée par les projections spatiales $\left(p r_{i}\right)_{i \in \Lambda}$ où:

$$
\begin{aligned}
& p r_{i}: \mathbb{R}^{\mathbb{Z}^{d}} \longrightarrow \mathbb{R} \\
& x=\left(x_{j}\right)_{j \in \mathbb{Z}^{d}} \longmapsto x_{i} .
\end{aligned}
$$

- $\mathcal{S}$ est l'espace des fonctions à décroissance rapide sur $\mathbb{Z}^{d}$. Son dual $\mathcal{S}^{\prime}$ est l'espace des fonctions à croissance lente, i.e. à croissance moins rapide que celle d'un polynôme.

Si l'on note, pour tout $r \in \mathbb{R},\|\cdot\|_{r}$ la norme dans l'espace de Hilbert à poids $\ell^{2}\left(\gamma^{(r)}\right)$ définie par: pour $x=\left(x_{i}\right)_{i \in \mathbb{Z}^{d}}$,

$$
\|x\|_{r}^{2}=\sum_{i \in \mathbb{Z}^{d}} \gamma_{i}^{(r)} x_{i}^{2},
$$

où les $\gamma_{i}^{(r)}$ sont les poids (polynomiaux si $r \in \mathbb{N}^{*}$ ) donnés par: $\gamma_{i}^{(r)}=(1+|i|)^{2 r}$ avec $|i|=\sum_{k=1}^{d}\left|i_{k}\right|$ pour $i=\left(i_{1}, \ldots, i_{d}\right) \in \mathbb{Z}^{d}$, alors on a:

$$
\begin{aligned}
\mathcal{S} & =\bigcap_{r \in \mathbb{N}^{*}}\left\{x \in \mathbb{R}^{\mathbb{Z}^{d}} ;\|x\|_{r}<\infty\right\} \text { et } \\
\mathcal{S}^{\prime} & =\bigcup_{r \in \mathbb{N}^{*}}\left\{x \in \mathbb{R}^{\mathbb{Z}^{d}} ;\|x\|_{-r}<\infty\right\} .
\end{aligned}
$$

Pour $r>0$, on note:

$$
E_{-r}=\left\{x \in \mathbb{R}^{\mathbb{Z}^{d}} ;\|x\|_{-r}<\infty\right\}
$$

qui est un espace de Hilbert séparable.

Sur un espace $\mathcal{X}$ normé on définit:

- $\mathcal{P}(\mathcal{X})$, l'ensemble des mesures de probabilité sur $\mathcal{X}$.

- $\mathcal{C}_{b}(\mathcal{X})$, l'ensemble des fonctions continues et bornées sur $\mathcal{X}$.

- $\operatorname{Lip}(\mathcal{X})$, l'ensemble des fonctions lipschitziennes sur $\mathcal{X}$ à valeurs réelles. 
Pour tout $f \in \operatorname{Lip}(\mathcal{X})$, on désigne par $[f]$ la constante de Lipschitz de $f \operatorname{sur} \mathcal{X}$, i.e.

$$
[f]=\sup _{\substack{x \neq y \\ x, y \in \mathcal{X}}} \frac{|f(x)-f(y)|}{\|x-y\|_{\mathcal{X}}} .
$$

Nous rappelons les hypothèses notées $\mathcal{H}_{0}$ d'existence et d'unicité de la solution de (1).

$\mathcal{H}_{0}\left\{\begin{array}{l}\alpha) \exists M \in \mathbb{R}, \forall i \in \mathbb{Z}^{d} \phi_{i}^{\prime \prime} \geq M \text { et } \sup _{i \in \mathbb{Z}^{d}}\left|\phi_{i}^{\prime}(0)\right|<\infty . \\ \beta) \exists\left(q_{i j}\right)_{i, j \in \mathbb{Z}^{d}} \text { positive avec } \sup _{i \in \mathbb{Z}^{d}} \sum_{j \neq i} q_{i j}<\infty \\ \text { telle que } \frac{\partial^{2}}{\partial x^{2}} \phi_{i, j}(x, y) \geq-q_{i j} \text { et } \sup _{i \in \mathbb{Z}^{d}} \sum_{j \neq i}\left|\phi_{i, j}^{\prime}(0,0)\right|<\infty . \\ \gamma) \exists\left(c_{i}\right)_{i} \in \mathcal{S} \text { telle que } \sup _{\substack{i, j \in \mathbb{Z}^{d} \\ i \neq j}}\left|\frac{\partial^{2}}{\partial x \partial y} \phi_{i, j}(x, y)\right| \leq c_{i-j} .\end{array}\right.$

Sous $\mathcal{H}_{0}$ (cf Doss \& Royer(1978), Shiga \& Shimizu(1980)), pour $x \in E_{-r}$, l'équation (1) a une unique solution forte markovienne à trajectoires continues dans $E_{-r}$.

On définit comme usuellement le semi-groupe de transition unitaire $\left(T_{t}\right)_{t \geq 0}$ associée à la solution $(X(t))_{t \geq 0}$ de $(1)$ par:

$\forall t \geq 0, x=\left(x_{i}\right)_{i \in \mathbb{Z}^{d}} \in E_{-r}$ et $f \in C_{b}\left(E_{-r}\right)$,

$$
T_{t} f(x)=E(f(X(t)) / X(0)=x) .
$$

\section{Propriétés ergodiques de la diffusion $(X(t))_{t \geq 0}$}

Nous rappelons d'abord la notion d'ergodicité d'un processus et ensuite le critère de Sunyach.

\subsection{Définition.}

La diffusion $(X(t))_{t \geq 0}$ solution de $(1)$ est dite ergodique dans $E_{-r}$ s'il existe une unique mesure de probabilité $\mu_{0}$ sur $E_{-r}$ (invariante sous l'action du semi-groupe de transition $T_{t}$ ) telle que: $\forall x \in E_{-r}$ et $f \in C_{b}\left(E_{-r}\right)$,

$$
\lim _{t \rightarrow \infty} T_{t} f(x)=\int_{E_{-r}} f d \mu_{0} .
$$


Remarque. L'ergodicité se teste sur les fonctions continues et bornées alors que dans le critère de Sunyach qui suit les fonctions tests sont les fonctions lipschitziennes. On rappelle (dans un contexte plus général) le critère de Sunyach (Sunyach (1975) Théorème 1) adapté au cas où le temps est continu.

Soit $(Y(t))_{t \geq 0}$ un processus de diffusion à valeurs dans un espace de Hilbert séparable $\mathcal{X}$ et $\left(P_{t}\right)_{t \geq 0}$ son semi-groupe de transition unitaire associé.

Proposition 1. Si le processus $(Y(t))_{t \geq 0}$ satisfait aux hypothèses suivantes:

i) $P_{t}$ est contractante $\operatorname{sur} \operatorname{Lip}(\mathcal{X})$, i.e. $\exists \lambda>0, \forall f \in \operatorname{Lip}(\mathcal{X})$

$$
\left[P_{t} f\right] \leq e^{-\lambda t}[f] \quad \forall t \geq 0,
$$

ii) $\exists y_{0} \in \mathcal{X}$ tel que $\forall t \geq 0$

$$
E\left(\left\|Y(t)-y_{0}\right\|_{\mathcal{X}}\right)<+\infty
$$

alors il existe une unique probabilité $\mu_{0}$ (invariante sous l'action de $P_{t}$ ) telle que pour tout $f \in \operatorname{Lip}(\mathcal{X})$ et $x \in \mathcal{X}$,

$$
\lim _{t \rightarrow \infty} T_{t} f(x)=\int_{\mathcal{X}} f d \mu_{0} .
$$

Nous allons montrer que la diffusion $(X(t))_{t \geq 0}$ solution de (1) satisfait ce critère dans $E_{-r}$ (défini par (2) et ensuite qu'elle est ergodique (pour cela on introduit la métrique de Vasserstein).

Le Théorème 1 qui suit est le résultat principal de ce travail.

Théorème 1. Considérons le système gradient stochastique (1) dans lequel l'interaction satisfait, en plus des hypothèses $\beta$ ) et $\gamma$ ) de $\mathcal{H}_{0}$, les hypothèses

$\left.\alpha^{\prime}\right)$ Uniformément en $i$, les $\phi_{i}$ sont strictement convexes, i.e. $\exists M>0$ $\forall i \in \mathbb{Z}^{d}, \phi_{i}^{\prime \prime}>M$ et $\sup _{i}\left|\phi_{i}^{\prime}(0)\right|<+\infty$.

ঠ) $\exists a=\left(a_{i}\right)_{i \in \mathbb{Z}^{d}} \in \mathcal{S}$, positive telle que $a_{0}=0, N=\sum_{k \neq 0} a_{k}<\infty$ et pour tous $x, y, z$, $t$ et $i, j \in \mathbb{Z}^{d}$,

$$
(x-y)\left(\phi_{i, j}^{\prime}(x, z)-\phi_{i, j}^{\prime}(y, t)\right) \geq a_{i-j}\left((x-y)^{2}-(z-t)^{2}\right) .
$$

7) $\exists r>0$ tel que:

$$
K(r)=\sup _{i \in \mathbb{Z}^{d}} \sum_{j \neq i} \frac{\gamma_{i}^{(r)}}{\gamma_{j}^{(r)}} a_{i-j}<M+N .
$$

Alors, $(X(t))_{t \geq 0}$ solution de (1) est ergodique dans $E_{-r}$. 
Remarque. Dans le cas où la portée de l'interaction est finie la suite $a=\left(a_{i}\right)_{i \in \mathbb{Z}^{d}}$ définie dans l'hypothèse $(\delta)$ a au plus un nombre fini de termes non nuls.

Démonstration du Théorème 1: Etant donné $x=\left(x_{i}\right)_{i \in \mathbb{Z}^{d}} \in E_{-r}$ (respectivement $y=\left(y_{i}\right)_{i \in \mathbb{Z}^{d}} \in E_{-r}$ ), notons $X^{x}(t)$ (resp. $X^{y}(t)$ ) la solution de (1) de condition initiale $x$ (resp. $y$ ) dans $E_{-r}$, on a: $\forall t \geq 0$, $i \in \mathbb{Z}^{d}$,

$$
\begin{aligned}
X_{i}^{x}(t) & -X_{i}^{y}(t)=\left(x_{i}-y_{i}\right)-\frac{1}{2} \int_{0}^{t}\left(\phi_{i}^{\prime}\left(X_{i}^{x}(s)\right)-\phi_{i}^{\prime}\left(X_{i}^{y}(s)\right)\right) d s \\
& -\frac{1}{2} \int_{0}^{t} \sum_{j \neq i}\left(\phi_{i, j}^{\prime}\left(X_{i}^{x}(s), X_{j}^{x}(s)\right)-\phi_{i, j}^{\prime}\left(X_{i}^{y}(s),\left(X_{j}^{y}(s)\right)\right) d s .\right.
\end{aligned}
$$

En dérivant $\left(X_{i}^{x}(t)-X_{i}^{y}(t)\right)^{2}$ par rapport à $t$, on a:

$$
\begin{aligned}
& \frac{d}{d t}\left(X_{i}^{x}(t)-X_{i}^{y}(t)\right)^{2}=-\left(X_{i}^{x}(t)-X_{i}^{y}(t)\right)\left(\phi_{i}^{\prime}\left(X_{i}^{x}(t)\right)-\phi_{i}^{\prime}\left(X_{i}^{y}(t)\right)\right) \\
& -\sum_{j \neq i}\left(X_{i}^{x}(t)-X_{i}^{y}(t)\right)\left(\phi_{i, j}^{\prime}\left(X_{i}^{x}(t), X_{j}^{x}(t)\right)-\phi_{i, j}^{\prime}\left(X_{i}^{y}(t), X_{j}^{y}(t)\right)\right) .
\end{aligned}
$$

D'après les assertions $\left(\alpha^{\prime}\right)$ et $(\delta)$ on a: $\forall t \geq 0, i \in \mathbb{Z}^{d}$

$$
\begin{aligned}
& \frac{d}{d t}\left(X_{i}^{x}(t)-X_{i}^{y}(t)\right)^{2} \\
& \quad \leq-(M+N)\left(X_{i}^{x}(t)-X_{i}^{y}(t)\right)^{2}+\sum_{j \neq i} a_{i-j}\left(X_{j}^{x}(t)-X_{j}^{y}(t)\right)^{2} .
\end{aligned}
$$

Posons: $\forall i, j \in \mathbb{Z}^{d}$,

$$
Q_{i, j}:= \begin{cases}-(M+N), & \text { si } i=j \\ a_{i-j}, & \text { si } i \neq j .\end{cases}
$$

Remarquons que $Q_{i, j}=Q_{j, i}$ car $\phi_{i, j}=\phi_{j, i}$ et donc pour tout $i \in \mathbb{Z}^{d}$, $a_{i}=a_{-i}$; on a donc $\forall i \in \mathbb{Z}^{d}$ et $t \geq 0$,

$$
\begin{aligned}
\frac{d}{d t}\left(X_{i}^{x}(t)-X_{i}^{y}(t)\right)^{2} & \leq \sum_{j \in \mathbb{Z}^{d}} Q_{i, j}\left(X_{j}^{x}(t)-X_{j}^{y}(t)\right)^{2} \\
\left(X_{i}^{x}(0)-X_{i}^{y}(0)\right)^{2} & =\left(x_{i}-y_{i}\right)^{2} .
\end{aligned}
$$

On a besoin maintenant d'un lemme de Gronwall infini-dimensionnel dans le cas où les constantes sont de signe quelconque (la "diagonale" $\left(Q_{i i}\right)_{i \in \mathbb{Z}^{d}}$ est négative). 
Lemme 1. Soient $\gamma=\left(\gamma_{i}\right)_{i \in \mathbb{Z}^{d}}$ et $a=\left(a_{i}\right)_{i \in \mathbb{Z}^{d}}$ des suites positives sur $\mathbb{Z}^{d}$ et $Q=\left(Q_{i, j}\right)_{i, j}$ une "matrice" sur $\mathbb{Z}^{d} \times \mathbb{Z}^{d}$, symétrique. Soit $u(t)=\left(u_{i}(t)\right)_{i \in \mathbb{Z}^{d}, t \geq 0}$ une suite de fonctions positives continues sur $\mathbb{R}_{+}$.

Supposons que:

(1) $a \in \ell^{1}(\gamma)$ et $\forall t \geq 0, \sup _{s \leq t} u(s) \in \ell^{1}(\gamma)$ i.e.

$$
\sum_{i \in \mathbb{Z}^{d}} \gamma_{i} a_{i}<\infty \text { et } \forall t \geq 0, \quad \sum_{i \in \mathbb{Z}^{d}} \gamma_{i} \sup _{s \leq t} u_{i}(s)<\infty
$$

(2) $\exists K \in \mathbb{R}, \forall j \in \mathbb{Z}^{d}$,

$$
\sum_{i \in \mathbb{Z}^{d}} \gamma_{i} Q_{i, j} \leq K \cdot \gamma_{j}
$$

Si $K \geq 0$, supposons que $\forall i \in \mathbb{Z}^{d}, t \geq 0$,

$$
u_{i}(t) \leq a_{i}+\sum_{j \in \mathbb{Z}^{d}} Q_{i, j} \int_{0}^{t} u_{j}(s) d s
$$

ou si $K<0$ supposons que la suite de fonctions $u$ est de plus dérivable sur $\mathbb{R}_{+}$et que $\forall i \in \mathbb{Z}^{d}, t \geq 0$

$$
u_{i}^{\prime}(t) \leq \sum_{j \in \mathbb{Z}^{d}} Q_{i, j} u_{j}(t), \quad u_{i}(0)=a_{i}
$$

Alors

$$
\sum_{i \in \mathbb{Z}^{d}} \gamma_{i} u_{i}(t) \leq e^{K t} \sum_{i \in \mathbb{Z}^{d}} \gamma_{i} a_{i}
$$

Remarque. L'inégalité (8) entraîne (7), donc faire l'hypothèse que (8) est vérifiée est plus fort qu'exiger que (7) le soit.

Démonstration du Lemme 1:

Cas $K \geq 0$ :

De l'inégalité (7) on a: $\forall t \geq 0$,

$$
\sum_{i \in \mathbb{Z}^{d}} \gamma_{i} u_{i}(t) \leq \sum_{i \in \mathbb{Z}^{d}} \gamma_{i} a_{i}=\int_{0}^{t} \sum_{i \in \mathbb{Z}^{d}} \sum_{j \in \mathbb{Z}^{d}} Q_{i, j} \gamma_{i} u_{j}(s) d s
$$


Par l'inégalité (6) et du fait que $Q_{i, j}$ est symétrique on a: $\forall t \geq 0$,

$$
\sum_{i \in \mathbb{Z}^{d}} \gamma_{i} u_{i}(t) \leq \sum_{i \in \mathbb{Z}^{d}} \gamma_{i} a_{i}+K \int_{0}^{t} \sum_{i \in \mathbb{Z}^{d}} \gamma_{i} u_{i}(s) d s .
$$

Posons, $v(t)=e^{-K t} \int_{0}^{t} \sum_{i \in \mathbb{Z}^{d}} \gamma_{i} u_{i}(s) d s$. La fonction $v$ est dérivable sur $\mathbb{R}_{+}$car $t \mapsto \sum_{i \in \mathbb{Z}^{d}} \gamma_{i} u_{i}(t)$ est continue sur $\mathbb{R}_{+}$. Sa dérivée vaut: $\sum_{i \in \mathbb{Z}^{d}} \gamma_{i} u_{i}(t)$, on a donc:

$$
\begin{aligned}
v^{\prime}(t) & =\left(\sum_{i \in \mathbb{Z}^{d}} \gamma_{i} u_{i}(t)-K \int_{0}^{t} \sum_{i \in \mathbb{Z}^{d}} \gamma_{i} u_{i}(s) d s\right) e^{-K t} \\
& \leq e^{-K t} \cdot \sum_{i \in \mathbb{Z}^{d}} \gamma_{i} a_{i}
\end{aligned}
$$

$$
\text { alors } \quad v(t) \leq \frac{1}{K} \cdot \sum_{i \in \mathbb{Z}^{d}} \gamma_{i} a_{i} \cdot\left(1-e^{-K t}\right)
$$

or $\int_{0}^{t} \sum_{i \in \mathbb{Z}^{d}} \gamma_{i} u_{i}(s) d s=e^{K t} v(t)$ donc,

$$
\begin{aligned}
\sum_{i \in \mathbb{Z}^{d}} \gamma_{i} u_{i}(t) & \leq \sum_{i \in \mathbb{Z}^{d}} \gamma_{i} a_{i}+\sum_{i \in \mathbb{Z}^{d}} \gamma_{i} a_{i} \cdot e^{K t}\left(1-e^{-K t}\right) \\
& =e^{K t} \cdot \sum_{i \in \mathbb{Z}^{d}} \gamma_{i} a_{i}
\end{aligned}
$$

ce qui nous donne l'inégalité (9) pour $K \geq 0$.

Cas où $K<0$ :

De l'inégalité (8) et par la symétrie de $Q_{i, j}$ on a: $\forall t \geq 0$,

$$
\begin{aligned}
& \sum_{i \in \mathbb{Z}^{d}} \gamma_{i} u_{i}^{\prime}(t) \leq K \sum_{i \in \mathbb{Z}^{d}} \gamma_{i} u_{i}(t) \\
& \sum_{i \in \mathbb{Z}^{d}} \gamma_{i} u_{i}(0)=\sum_{i \in \mathbb{Z}^{d}} \gamma_{i} a_{i} .
\end{aligned}
$$

Posons

$$
m(t)=\sum_{i \in \mathbb{Z}^{d}} \gamma_{i} u_{i}(t) \text { et } g(t)=e^{-K t} m(t)
$$

on a:

$$
\begin{aligned}
g^{\prime}(t) & =\left(m^{\prime}(t)-K m(t)\right) e^{-K t} \\
& \leq 0
\end{aligned}
$$


La fonction $g$ est donc décroissante sur $\mathbb{R}_{+}$. Donc $\forall t \geq 0$,

$$
g(t) \leq \sum_{i \in \mathbb{Z}^{d}} \gamma_{i} a_{i}=g(0)
$$

D'où l'inégalité (9) pour $K<0$.

Remarque. Le choix judicieux de la constante $K$ du Lemme 1 est donné par

$$
\begin{aligned}
K & =\sup _{j \in \mathbb{Z}^{d}} \sum_{i \in \mathbb{Z}^{d}} \frac{\gamma_{i}}{\gamma_{j}} Q_{i, j} \\
& =\sup _{j \in \mathbb{Z}^{d}} \sum_{i \in \mathbb{Z}^{d}} \frac{\gamma_{j}}{\gamma_{i}} Q_{i, j}
\end{aligned}
$$

par la symétrie de $Q_{i, j}$.

Suite de la démonstration du Théorème 1: La suite de fonctions $u_{i}(t)=$ $\left(X_{i}^{x}(t)-X_{i}^{y}(t)\right)^{2}$ satisfait les hypothèses du Lemme 1. En particulier, par construction même de la solution de $(1),\left(\sup _{s \leq t} u_{i}(s)\right)_{i} \in \ell^{1}\left(\gamma^{(r)}\right)$ (cf Shiga \& Shimizu (1980)). D'où, grâce au Lemme 1,

(10) $\sum_{i \in \mathbb{Z}^{d}} \gamma_{i}^{(r)}\left(X_{i}^{x}(t)-X_{i}^{y}(t)\right)^{2}$

$$
\leq \exp ((K(r)-M-N) t) \cdot \sum_{i \in \mathbb{Z}^{d}} \gamma_{i}^{(r)}\left(x_{i}-y_{i}\right)^{2}
$$

Soient $f \in \operatorname{Lip}\left(E_{-r}\right)$ et $t \geq 0$

$$
\begin{aligned}
\left|T_{t} f(x)-T_{t} f(y)\right| & =\left|E\left(f\left(X^{x}(t)\right)-f\left(X^{y}(t)\right)\right)\right| \\
& \leq E\left|f\left(X^{x}(t)\right)-f\left(X^{y}(t)\right)\right| \\
& \leq\left[E\left|f\left(X^{x}(t)\right)-f\left(X^{y}(t)\right)\right|^{2}\right]^{1 / 2} \\
& \leq[f] \cdot\left(E\left\|X^{x}(t)-X^{y}(t)\right\|_{-r}^{2}\right)^{1 / 2}
\end{aligned}
$$

En combinant les inégalités (10) et (11), on a: $\forall t \geq 0$,

$$
\left|T_{t} f(x)-T_{t} f(y)\right| \leq[f] \cdot \exp \left(\frac{K(r)-M-N}{2} \cdot t\right) \cdot\|x-y\|_{-r} .
$$


Puisque, par l'hypothèse $(\eta)$ la constante $K(r)-M-N$ est strictement négative, la diffusion $(X(t))_{t \geq 0}$ perd avec une vitesse exponentielle, la mémoire de son état initial. On a donc:

$$
\begin{aligned}
& \forall t \geq 0 \text { et } f \in \operatorname{Lip}\left(E_{-r}\right), \\
& \left.\qquad T_{t} f\right] \leq[f] \cdot \exp \left(\frac{K(r)-M-N}{2} \cdot t\right) .
\end{aligned}
$$

Donc l'hypothèse i) de la Proposition 1 est satisfaite.

D'autre part, par construction (cf Shiga \& Shimizu (1980)), le processus de diffusion $(X(t))_{t \geq 0}$ satisfait pour tout $t \geq 0, E\|X(t)\|_{-r}<+\infty$. Donc l'hypothèse (ii) de la Proposition 1 est satisfaite et cela prouve que la diffusion $(X(t))_{t \geq 0}$ admet une unique probabilité $\mu_{0}$, invariante sous l'action de $T_{t}$, telle que: $\forall f \in \operatorname{Lip}\left(E_{-r}\right)$ et $x \in E_{-r}$,

$$
\lim _{t \rightarrow \infty} T_{t} f(x)=\int_{E_{-r}} f d \mu_{0}
$$

Nous allons montrer que cette conclusion reste vraie pour les fonctions $f$ continues bornées sur $E_{-r}$. Soit

$$
\begin{aligned}
\mathcal{P}_{1}\left(E_{-r}\right):=\left\{P \in \mathcal{P}\left(E_{-r}\right)\right. \text { tel que } & \\
& \left.\quad \exists x_{0} \in E_{-r}, \int_{E_{-r}}\left\|x-x_{0}\right\|_{-r} d P(x)<+\infty\right\} .
\end{aligned}
$$

Munissons l'espace $\mathcal{P}_{1}\left(E_{-r}\right)$ de la métrique de Vasserstein, notée $\mathbf{R}$, définie par: pour $P, Q \in \mathcal{P}\left(E_{-r}\right)$,

$$
\mathbf{R}(P, Q):=\inf _{X, Y} E\|X-Y\|_{-r},
$$

où $X$ (respectivement $Y$ ) parcourt l'ensemble des variables aléatoires à valeurs dans $E_{-r}$ de loi $P$ (resp. $Q$ ).

Cette définition de $\mathbf{R}$ est équivalente à la relation duale de Kantorovich: plus précisément, pour $P, Q \in \mathcal{P}_{1}\left(E_{-r}\right)$,

$$
\mathbf{R}(P, Q)=\sup _{[f] \leq 1}\left|\int_{E_{-r}} f d(P-Q)\right| .
$$

L'espace $\left(\mathcal{P}_{1}\left(E_{-r}\right), \mathbf{R}\right)$ est un espace métrique complet séparable et la convergence d'une suite $\left(P_{n}\right)_{n}$ de $\mathcal{P}_{1}\left(E_{-r}\right)$ au sens de la métrique $\mathbf{R}$ est équivalente à la convergence étroite de $\left(P_{n}\right)_{n}$ (voir Dudley $(1976)$, Theorem 8.3). Ainsi pour tout $f \in C_{b}\left(E_{-r}\right)$ et $x \in E_{-r}, \lim _{t \rightarrow \infty} T_{t} f(x)=$ $\int_{E_{-r}} f d \mu_{0}$, où $\mu_{0}$ est la probabilité invariante sous l'action de $T_{t}$. C'est l'ergodicité de la diffusion $(X(t))_{t \geq 0}$ solution de (1). 


\section{Exemples}

\subsection{Un cas d'interaction à portée finie.}

On considère l'hamiltonien $h=\left(h_{i}\right)_{i \in \mathbb{Z}^{d}}$ donné au site $i \in \mathbb{Z}^{d}$ par

$$
h_{i}(x)=V\left(x_{i}\right)+\frac{1}{2} \sum_{|i-j| \leq \rho}\left(x_{i}-x_{j}\right)^{2}
$$

où $\left.x=\left(x_{j}\right)_{j \in \mathbb{Z}^{d}} \in \mathbb{R}^{\mathbb{Z}^{d}}, \rho \in\right] 0,+\infty[$ est la portée de l'interaction et $V \in C^{2}(\mathbb{R})$.

L'interaction par paire $-x_{i} x_{j}$ est quadratique pour $i$ et $j$ tels que $|i-j| \leq \rho$.

De la formule (12), on peut définir les potentiels d'interaction $\left(\phi_{i}\right)_{i}$ et $\left(\phi_{i, j}\right)_{i, j}$ par: $\forall i, j \in \mathbb{Z}^{d}$,

$$
\phi_{i} \equiv V \text { et } \phi_{i, j}\left(x_{i}, x_{j}\right)=\frac{1}{2}\left(x_{i}-x_{j}\right)^{2} \text { avec } x_{i}, x_{j} \in \mathbb{R} .
$$

Posons: pour $i \in \mathbb{Z}^{d}$ et $x=\left(x_{i}\right)_{i \in \mathbb{Z}^{d}} \in \mathbb{R}^{\mathbb{Z}^{d}}$,

$$
b_{i}^{(1)}(x):=-\frac{1}{2} \frac{\partial}{\partial x_{i}} h_{i}(x)=-\frac{1}{2}\left(V^{\prime}\left(x_{i}\right)+\sum_{|i-j| \leq \rho}\left(x_{i}-x_{j}\right)\right) .
$$

Considérons l'équation différentielle stochastique suivante associée à $h=\left(h_{i}\right)_{i \in \mathbb{Z}^{d}}: \forall i \in \mathbb{Z}^{d}$,

$$
\begin{aligned}
d X_{i}^{(1)}(t) & =d B_{i}(t)+b_{i}^{(1)}\left(X^{(1)}(t)\right) d t, \quad t>0 \\
X_{i}^{(1)}(0) & =x_{i}^{(1)} \in \mathbb{R} .
\end{aligned}
$$

Proposition 2. Si le potentiel d'interaction propre $V$ est strictement convexe alors il existe $r>0$ tel que l'équation (13) ait une unique solution markovienne, notée $\left(X^{(1)}(t)\right)_{t \geq 0}$, qui est ergodique dans $E_{-r}$ (défini en (2)).

Démonstration: Puisque $V$ est strictement convexe et que $\frac{\partial^{2}}{\partial x \partial y} \phi_{i, j}(x, y)=-1$, alors les hypothèses $\alpha, \beta, \gamma$ de $\mathcal{H}_{0}$ sont satisfaites.

Par la stricte convexité de $V$, il existe $M>0$ tel que l'hypothèse $\left(\alpha^{\prime}\right)$ du Théorème 1 soit satisfaite. D'autre part, pour tous réels $x, y, z, t$ et pour $i, j \in \mathbb{Z}^{d}$, on a:

$$
\begin{aligned}
(x-y)\left(\phi_{i, j}^{\prime}(x, z)-\phi_{i, j}^{\prime}(y, t)\right) & =(x-y)^{2}-(x-y)(z-t) \\
& \geq \frac{1}{2}\left((x-y)^{2}-(z-t)^{2}\right) .
\end{aligned}
$$


Ainsi l'hypothèse $(\delta)$ du Théorème 1 est vérifiée avec

$$
a_{i}= \begin{cases}\frac{1}{2} & \text { si }|i| \leq \rho \\ 0 & \text { sinon }\end{cases}
$$

Donc:

$$
N=\sum_{0<|k| \leq \rho} a_{k}=\sum_{k \in B_{\rho}(0) \backslash\{0\}} \frac{1}{2}=\frac{1}{2}\left(\left|B_{\rho}(0)\right|-1\right),
$$

où $B_{\rho}(i)=\left\{j \in \mathbb{Z}^{d} ;|i-j| \leq \rho\right\}$ et $\left|B_{\rho}(0)\right|$ est le cardinal de $B_{\rho}(0)$.

Il nous reste à montrer l'existence de $r \in \mathbb{Q}_{+}^{*}$ tel que l'hypothèse $(\eta)$ du Théorème 1 soit satisfaite.

$$
\begin{aligned}
K(r) & =\sup _{i \in \mathbb{Z}^{d}} \sum_{0<|j-i| \leq \rho}\left(\frac{1+|i|}{1+|j|}\right)^{2 r} \cdot \frac{1}{2} \\
& =\sup _{i \in \mathbb{Z}^{d}} \sum_{0<|k| \leq \rho}\left(\frac{1+|i|}{1+|i+k|}\right)^{2 r} \cdot \frac{1}{2} .
\end{aligned}
$$

Par l'inégalité élémentaire: $|i|-|k| \leq|i+k|$, on a:

$$
\begin{aligned}
K(r) & =\sup _{i \in \mathbb{Z}^{d}} \sum_{k \in B_{\rho}(0) \backslash\{0\}}\left(\frac{1+|i+k|+|k|}{1+|i+k|}\right)^{2 r} \cdot \frac{1}{2} \\
& \leq \sum_{k \in B_{\rho}(0) \backslash\{0\}}(1+|k|)^{2 r} \cdot \frac{1}{2} \\
& \leq(1+\rho)^{2 r} \cdot \frac{1}{2}\left(\left|B_{\rho}(0)\right|-1\right) .
\end{aligned}
$$

On peut toujours choisir $r>0$ suffisamment petit pour que:

$$
1<(1+\rho)^{2 r}<1+\frac{2 M}{\left|B_{\rho}(0)\right|-1} .
$$

Donc l'hypothèse $(\eta)$ du Théorème 1 est satisfaite.

Remarque. Pour $\rho=1$ (interaction au plus proche voisin) le système gradient stochastique (13) a été étudié par Royer (cf Royer (1979)). Il a montré l'existence d'une unique mesure de probabilité (de Gibbs) invariante mais n'a pas explicité la convergence vers cette mesure de Gibbs. 


\subsection{Un exemple typique d'interaction à portée infinie.}

Soient donnés $a=\left(a_{i}\right)_{i \in \mathbb{Z}^{d}}$ une suite positive de $\mathcal{S}$ avec $a_{0}=0$ et $N=\sum_{k \neq 0} a_{k}<\infty$, et une fonction $V \in \mathcal{C}^{2}(\mathbb{R})$.

Considérons l'hamiltonien $h=\left(h_{j}\right)_{j \in \mathbb{Z}^{d}}$ donné au site $i \in \mathbb{Z}^{d}$ par:

$$
h_{i}(x)=V\left(x_{i}\right)+\sum_{j \neq i} a_{i-j}\left(x_{i}-x_{j}\right)^{2}, \quad x=\left(x_{i}\right)_{i \in \mathbb{Z}^{d}} .
$$

Dans cet exemple, l'interaction par paire est modélisée par: $-2 a_{i-j} x_{i} x_{j}$ $(i \neq j)$, et la portée de l'interaction n'est pas a priori finie. Pour $i, j \in \mathbb{Z}^{d}$ on peut définir les potentiels d'interaction $\phi_{i}$ et $\phi_{i, j}$ par:

$$
\phi_{i} \equiv V \text { et } \phi_{i, j}\left(x_{i}, x_{j}\right)=\left(x_{i}-x_{j}\right)^{2}, \quad x_{i}, x_{j} \in \mathbb{R} .
$$

On pose: $\forall i \in \mathbb{Z}^{d}$ et $x=\left(x_{j}\right)_{j \in \mathbb{Z}^{d}}$,

$$
b_{i}^{(2)}(x):=-\frac{1}{2} \frac{\partial}{\partial x_{i}} h_{i}(x)=-\frac{1}{2}\left(V^{\prime}\left(x_{i}\right)+\sum_{j \neq i} 2 a_{i-j}\left(x_{i}-x_{j}\right)\right) .
$$

Considérons le système gradient stochastique associé à l'hamiltonien défini en (14) suivant: $\forall i \in \mathbb{Z}^{d}$,

$$
\begin{aligned}
d X_{i}^{(2)}(t) & =d B_{i}(t)+b_{i}^{(2)}\left(X^{(2)}(t)\right) d t, \quad t>0 \\
X_{i}^{(2)}(0) & =x_{i}^{(2)} \in \mathbb{R} .
\end{aligned}
$$

Proposition 3. Si le potentiel d'interaction propre $V$ introduit en (14) est strictement convexe alors la solution $\left(X^{(2)}(t)\right)_{t \geq 0}$ de l'équation (15) est ergodique dans un espace $E_{-r}, r \in \mathbb{Q}^{+}$.

Démonstration: Puisque la fonction $V$ est strictement convexe et la suite $a=\left(a_{i}\right)_{i \in \mathbb{Z}^{d}}$ (donnée dans (14)) appartient à $\mathcal{S}$, les hypothèses $\alpha$, $\beta$ et $\gamma$ de $\mathcal{H}_{0}$ sont bien satisfaites.

Par la stricte convexité de $V$, il existe $M>0$ tel que l'hypothèse $\left(\alpha^{\prime}\right)$ du Théorème 1 soit vérifiée. L'assertion $(\delta)$ est aussi satisfaite avec $a=\left(a_{i}\right)_{i \in \mathbb{Z}^{d}}$ défini en (14). Il nous reste à vérifier $(\eta)$. On sait que

$$
K(r)=\sup _{i \in \mathbb{Z}^{d}} \sum_{j \neq i}\left(\frac{1+|i|}{1+|j|}\right)^{2 r} a_{i-j}
$$


En utilisant l'inégalité élémentaire: $|i|-|j| \leq|i-j|$ on a:

$$
\begin{aligned}
K(r) & \leq \sup _{i \in \mathbb{Z}^{d}} \sum_{j \neq i}\left(\frac{1+|j|+|i-j|}{1+|j|}\right)^{2 r} a_{i-j} \\
& \leq \sum_{k \neq 0}(1+|k|)^{2 r} a_{k}=\bar{K}(r) .
\end{aligned}
$$

Puisque $a=\left(a_{i}\right)_{i \in \mathbb{Z}^{d}} \in \mathcal{S}$, elle décroît plus vite que tout polynôme, en particulier que $(1+|i|)^{-2(r+p)}$ avec $p>\frac{d}{2}$. Ce qui fait de $\bar{K}(r)$ une somme de série de fonctions normalement convergentes. Donc la fonction $\bar{K}$ est continue en $r \geq 0$ avec $\bar{K}(0)=N$. On peut donc toujours choisir $r>0$ (suffisamment petit) pour lequel $K(r)<M+N$. Donc l'hypothèse $(\eta)$ du Théorème 1 est satisfaite.

Remerciements. Nous remercions vivement G. Royer pour les différentes discussions que nous avons eues avec lui, très utiles pour l'accomplissement de ce travail.

\section{Références}

1. S. Albeverio, Y. G. Kondratiev et M. Röckner, Ergodicity of $L^{2}$-semi-groups and extremality of Gibbs states, J. Funct. Anal. 144 (1997), 394-423.

2. G. DA PRAto Et J. ZABCZYK, Convergence to equilibrium for classical and quantum spin systems, Probab. Theory Related Fields 103 (1995), 529-552.

3. H. Doss et G. Royer, Processus de diffusion associé aux mesures de Gibbs sur $\mathbb{R}^{\mathbb{Z}^{d}}$, Z. Wahrs. verw. Geb. 46 (1978), 107-124.

4. R. Dudley, "Prob. and metrics," Aarhus lecture notes, 1976.

5. G. Royer, Processus de diffusion associé à certaines modèles d'Ising à spins continus, Z. Wahrs. verw. Geb. 46 (1979), 165-176.

6. T. Shiga ET A. Shimizu, Infinite dimensional stochastic differential equations and their applications, J. Math. Kyoto Univ. 20(3) (1980), 395-416.

7. C. Sunyach, Une classe de chaînes de Markov récurrentes sur un espace métrique complet, Ann. Inst. H. Poincaré XI(4) (1975), 325-343. 
8. B. Zegarlinski, The strong decay to equilibrium for the stochastic dynamics of unbounded spin systems on a lattice, Comm. Math. Phys. 175 (1996), 401-432.

\author{
Laboratoire de Statistique et Probabilités \\ EP CNRS 1765, Bât. M2 \\ Université Lille 1 \\ F-59655 Villeneuve d'Ascq Cedex \\ FRANCE \\ e-mail: Sylvie.Roelly@univ-lille1.fr
}

Primera versió rebuda el 17 d'abril de 1998,

darrera versió rebuda el 31 d'agost de 1998 